A N N A L E S Annales de Bretagne et des Pays de l'Ouest

\title{
La recherche à l'université du Maine (Le Mans) de 1998 à 2003
}

\section{(2) OpenEdition}

Journals

Édition électronique

URL : http://journals.openedition.org/abpo/1293

DOI : 10.4000/abpo.1293

ISBN : 978-2-7535-1494-2

ISSN : 2108-6443

\section{Éditeur}

Presses universitaires de Rennes

\section{Édition imprimée}

Date de publication : 20 juillet 2004

Pagination : 183-197

ISBN : 978-2-7535-0031-0

ISSN : 0399-0826

\section{Référence électronique}

"La recherche à l'université du Maine (Le Mans) de 1998 à 2003 ", Annales de Bretagne et des Pays de l'Ouest [En ligne], 111-2 | 2004, mis en ligne le 20 juillet 2006, consulté le 15 septembre 2020. URL : http://journals.openedition.org/abpo/1293 


\section{La recherche à l'université du Maine (Le Mans) de 1998 à 2003}

\section{Thèses}

\section{4}

\section{Histoire médiévale}

BerTIAUX, Marie-Cécile, Les résidences des évêques de Troyes dans leur diocèse à la fin du Moyen Âge, dir. A. RENOUX.

\section{3}

\section{Histoire moderne}

AUDIBERT, David, Épiciers de l'Ouest. Le Mans, Angers, Nantes au XVIII ${ }^{e}$ siècle. Étude comparative, dir. A. FILLON.

\section{2}

\section{Histoire moderne}

HeICHETTE, Michel, Sociabilité et sensibilités collectives au XVIIr siècle. L'exemple du pays sabolien, dir. J.-M. CONSTANT.

\section{1}

\section{Histoire moderne}

BARILLY-LEGUY, Martine, Une famille mancelle du grand siècle : les Bodreau et leur livre (1567-1675), dir. M. MÉNARD.

\section{9}

Histoire médiévale

LEPAREUR, Florence, La vie paroissiale dans le Haut Maine à la fin du Moyen Âge (1367-1530) d'après les archives fabriciales, dir. A. RENOUX.

CASSET, Marie, Les résidences rurales et semi-rurales des archevêques et évêques normands au Moyen Âge, dir. A. RENOUX.

\section{Histoire moderne}

PIFfRE, Véronique, Pauvreté dans le Haut Maine : charité, assistance et lutte contre la marginalité (1650-1789), dir. A. FILLON.

\section{8}

\section{Histoire grecque}

THIRY, Sébastien, Les îles ioniennes de 480 à 167 avant J.-C., étude de géopolitique, dir. Patrice BRUN.

\section{Histoire romaine}

GulLLEUX, Joseph, L'enceinte gallo-romaine du Mans, dir. J. BIARNE.

Diplômes d'Études Approfondies (DEA)

2003

\section{Histoire médiévale}

LeProvost, Céline, Les matériaux de maçonnerie des fortifications du HautMaine du Xfe au XII siècle, dir. A. RENOUX.

\section{Histoire moderne}

Poisson, Guillaume, Les Choiseul aux Affaires étrangères (1758-1770). Parentèle, amitié et fidélité, dir. J.-M. CONSTANT.

LHôPITAL, Claire-Séverine, Une famille noble du Haut-Maine dans la tourmente révolutionnaire, les Bastard de Fontenay, 1770-1830, dir. J.-M. CONSTANT GÉNEAU, Catherine, Des racines des chênes à la cime de l'érable. L'émigration perche-

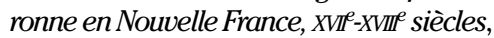
dir. J.-M. CONSTANT et L. BOURQUIN.

SANCHEZ-RodriguEZ, Anna-Maria, Les groupes privilégiés dans la société rurale d'Ancien régime. Essai de com- 
paraison des patrimoines fonciers de la noblesse européenne, dir. J.-M. CONSTANT.

SUZANNE, Jean-François, Les municipalités des villes du Perche dans la seconde moitié du XVIII siècle, dir. L. BOURQUIN.

LE Touzé-Derobert, Isabelle, Foi et Fidélité : les nobles bas-normands à l'heure des choix (vers 1500-1685), dir. Laurent BOURQUIN.

\section{Histoire contemporaine}

PAGEOT, Alexandra, Les femmes dans le monde rural en Sarthe entre 1950 et 1960, dir. N. VIVIER.

Rigollet, Laurent, Mouvements et convergences solidaires dans la Sarthe en crise, dir. C. MANIGAND.

ANFRAY, Maggy, L'émancipation des femmes à travers la presse sarthoise de l'entre-deux guerres, dir. N. VIVIER.

\section{2}

\section{Histoire grecque}

BOURROUX, Antoine, Relations d'hospitalité, réseaux d'alliances privées et diplomatie en Grèce ancienne, dir. A. TOURRAIX.

\section{Histoire romaine}

LOISEAU, Christophe, Le métal dans la construction des monuments publics; l'exemple des sanctuaires d'époque gallo-romaine, dir. M.-C. L'HUILLIER.

CHAuvin, Jean-Pierre, L'érudition mayennaise, dir. M.-C. L'HUILLIER et B. WACHÉ,

\section{Histoire médiévale}

DOUMERC, François, Les réseaux sociopolitiques des Rorgonides dans le monde franc (début $\mathrm{VIII}^{e}$-début XI siècles), dir. A. RENOUX.

AlBERT, Céline, Le calcaire dans la construction des églises médiévales $d u$ doyenné de Vallon (Sarthe), $X^{e}-X v^{e}$ siècles, dir. A. Renoux.

\section{Histoire moderne}

CotTeret, Betty, Prisons et châtiments au Mans au XVIII ${ }^{e}$ siècle, dir. L. BOURQUIN.
GERMOND, Stéphanie, Entre Beauce et Perche, la vie dans les fermes des laboureurs dans la Baronnie de Courville au XVIII siècle, dir. J.-M. CONSTANT.

\section{Histoire contemporaine}

LÉANDRO DE MÉDEIROS, Riccardo, Le feuilleton radiophonique : de la salle de séjour au magasin (exemple de Florianopolis-Brésil), dir. B. WACHÉ.

АнмAт, Mahamat Yacoub, Les relations franco-tchadiennes dans les années 1960, dir. B. WaCHÉ.

COLLET, Joakim, La Chouannerie dans la Mayenne, dir. B. WACHÉ.

Deulofeu, Nicolas, Foires et marchés des départements de la Sarthe et de la Mayenne, dir. N. VIVIER.

ESNEAULT, Mathias, La reconstruction en Normandie (1946-1954), dir. N. VIVIER.

Delozier, Linda, La déviance des femmes en France et en Angleterre à la fin $d u X I x^{e}$ siècle. L'exemple des Ornaises et des femmes vivant dans le Kent entre 1880 et 1901, dir. N. VIVIER.

HERVouËT, Thomas, Pour une étude de l'oraison chrétienne au $X X^{e}$ siècle, dir. B. Waché.

Bouland, Magali, Le petit commerce dans la Sarthe au XIXe siècle. Le boucher et la boutique, dir. N. VIVIER.

\section{1}

\section{Histoire ancienne}

DuFEu, Laurent, Les sophistes et le Politique dans la cité grecque, dir. A. TOURRAIX.

\section{Histoire médiévale}

MASNIÈRE-BUREAU, Isabelle, Le réseau des villes secondaires dans le Maine au Moyen Âge, dir. A. RENOuX.

BOUVIER, Ludovig, Les femmes dans le théâtre comique de la fin du Moyen Agge, dir. A. RENOUX.

\section{Histoire moderne}

Coupé, Cyril, Les petits hôpitaux du HautMaine et de l'Anjou fléchois, dir. J.-M. CONSTANT. 
Julien, Alexandre, La Société bourgeoise aux confins du Maine et du Perche. Modes de vie de sociabilité dans la France moderne (XVI-XVIII siècle), dir. L. BOURQUIN.

NAVAR, Julien, Les laboureurs du Saonois au XVIII $I^{e}$ siècle, dir. L. BOURQUIN.

FORTIER, Jérôme, Les justices seigneuriales dans le Haut-Maine. L'exemple de la baronnie de Bonnétable, dir. L. BOURQUIN.

Sybille, Christine, Le Vieux Mans au fil du temps, dir. J.-M. CONSTANT.

SALAÜN, Marion, La vie spirituelle à l'abbaye Saint-Florent à Saumur (15371610), dir. L. BOURQUIN.

GAUCHER, Aurélia, Catalogue de la collection Godard d'Alençon, dir. A. DUPRAT.

BouvET, Valérie, Bibliographie raisonnée de l'imagerie populaire, dir. A. DUPRAT.

\section{Histoire contemporaine}

MOURAHIB, Bidar, L'évolution de la vision de l'Islam à travers Ouest-Éclair et Ouest-France (1899-1999), dir. B. WACHÉ.

NiColle, David, Le pays manceau à l'heure allemande (juin 1940-août 1944), évolution des mentalités, profiteurs de guerre et collaboration, dir. B. WACHÉ.

Barbier, Élise, Le Clergé sarthois pendant la Révolution française : l'exemple du district de La Flèche, dir. B. WaCHÉ.

Duval, Angélique, Retombées des lois anti-congréganistes dans la Sarthe (1880-1914), dir. B. WACHÉ.

BORDIER, Christophe, Les comices agricoles d'Eure-et-Loir (1835-1898), dir. N. VIVIER.

TeSBER, Andréa, La lecture de la Bande Dessinée et de lectorat manceau aujourd'hui, dir. A. DUPRAT.

TRÉOL, Mickaël, La Reconstruction de l'agriculture dans le département de la Manche (1919-1930), dir. N. VIVIER.

Ligneul, Jérôme, La diffusion de l'épargne grâce à la propagande, aux caisses d'épargne scolaires et aux succursales, dir. N. VIVIER.

\section{9}

\section{Histoire ancienne}

FERNANDEZ, Éric, La forêt dans l'Ouest de la Gaule ( ${ }^{e r}$-VIe siècle) : l'exemple de la Sarthe, dir. J. BIARNE.

GaIGNon, Maryse, Les Dieux et les figures de la famille impériale, de Valérien à Gallien d'après les monnaies, dir. J. BIARNE.

\section{Histoire médiévale}

ChATELAIN, Hélène, Le mobilier royal et princier au XIV siècle d'après les inventaires publiés, dir. A. RENOUX.

GASCHET, Valérie, La musique au Mans à la fin du Moyen Âge, dir. A. Renoux.

Oudin, Frédéric, La seigneurie de La Guierche (XI'-début XVI siècle), dir. A. RENOUX.

PIRON, Céline, La place de l'Église du Pré dans l'art roman manceau : historiographie, architecture et problématiques, dir. A. RENOUX.

\section{Histoire moderne}

AUDIBERT, David, Épiciers du Mans et d'Angers dans la $2^{e}$ moitié $d u$ XVII e siècle - étude comparative, dir. A. FILLON.

DEVREKER, Sabrina, Marchands de l'habillement et de la parure au Mans au XVIII $e^{e}$ siècle, dir. A. FILLON.

Duval, Romain, La légende du "dict des trois morts et des trois vifs "dans la peinture murale du Maine au $X V^{e}$ et au XVI siècle, dir. M. MENARD.

EudckMAns, Natacha, Armées et Noblesses protestantes dans le centre-ouest de la France pendant les guerres de religion, dir. J.-M. CONSTANT.

GARREAU, Renold, L'administration municipale alençonnaise à l'époque révolutionnaire (1789-1799), dir. A. FILLON.

GuERnEve, Sonia, Les artisans du cuir au Mans au XVIII siècle, dir. A. FILLON.

RAVALET, Richard, Les élites urbaines à Angers au XVI siècle, dir. J.-M. CONSTANT.

Salmon, David, Les gentilshommes dans l'élection d'Alençon XVII -XVIII ${ }^{e}$ siècle, dir. J.-M. CONSTANT. 


\section{Histoire contemporaine}

COCHONNEAU-CORDIER, Gilles, Jean Gauchon, "le seul avocat ", dir. B. WACHÉ.

DELAPEYRIÈre-Delalande, Marie-José, $L a$ société théosophique - La théosophie 1900-1925, dir. B. WACHÉ.

Demeule, Fabienne, Les sociétés de secours mutuels dans la Sarthe, dir. B. WaCHÉ.

FERRÉ, Yoannick, Les sociétés hippiques de la Sarthe au XIX ${ }^{e}$ siècle, dir. N. VIVIER.

JOUBERT, Séverine, Agriculture et chemins de fer dans l'Orne $-2^{e}$ moitié $d u$ $X I X X^{e}$ siècle, dir. N. VIVIER.

LouIs, Axelle, Autour de Robert Triger 1856-1927, dir. B. WaCHÉ.

PaInEAU, Rémi, Les débuts de Paris Match 1949-1955, dir. B. WACHÉ.

\section{8}

\section{Histoire ancienne}

FAUQuier, Michel, Dieu ou César, idéal de sainteté et service de la cité en Gaule au ve et vi siècle, dir. J. BIARNE.

\section{Histoire médiévale}

Nevoux, Gaël, La châtellerie de Sillé-leGuillaume du X $P^{e}$ au $X v^{e}$ siècle, dir. A. RENOUX.

PoIGNANT, Laurence, Les formes anthoponyniques et leur évolution dans le Maine du Moyen Âge central d'après les données des cartulaires de Perseigne et de Vivoin, dir. A. RENOuX.

TouBoulic, Nathalie, Topographie et architecture des abbayes et prieurés $d u$ Bas-Maine médiéval, dir. A. RENOUX.

\section{Histoire moderne}

BoUlAY, Frédéric, Apothicaires aux XVII et XVII $e^{e}$ siècles dans les limites actuelles du département de la Sarthe, dir. J.-M. CONSTANT.

BRY-LENOIR, Claire, Les notables mortagnais du XVIII siècle, dir. A. FILLON.

Foulard, Damien, Métiers de l'eau sur les bassins du Loir et de la Sarthe dans l'Anjou et le Maine au XVIII siècle, dir. A. FILLON.

PEIGNÉ, Alain, Attitudes nobiliaires et état d'esprit de la famille de Rians de
Villeray (XVI'-XVII siècle), dir. J.-M. CONSTANT.

LEFUSTEC, Marie-Annick, Les monographies communales rédigées par les instituteurs de la Mayenne en 1889, dir. A. FILLON)

\section{Histoire contemporaine}

JouAN, Gwennaelle, Les religieuses qui soignent en Sarthe au XIX $X^{e}$ siècle, dir. B. WaCHÉ.

GRANGER, Tiphaine, Entre illégalité et légitimité : l'IRA provisoire de 1969 à 1994, dir. B. WACHÉ.

GUYARD, Armelle, L'action sociale et politique des catholiques du Maine dans l'entre deux-guerres, dir. B. WACHÉ.

\section{Mémoires de Maîtrise}

\section{3}

\section{Histoire ancienne}

CACHAN, Miguel, La magie dans l'Antiquité romaine à travers l'œuvre d'Apulée, dir. M.-C. L'HuILLIER.

CHASLES, Erwan, Les femmes dans la société gallo-romaine. Étude des monuments funéraires, dir. E. BERTRAND.

EDON, Christelle, La place de l'enfant dans la peinture pompéienne, dir. E. BERTRAND.

LEMAîTRE, Marion, Les femmes dans la Rome mythique et archaïque, dir. A. AlLELY.

MARTIN, François, Les déclarations d'Hostis publicis dans la Rome républicaine, dir. A. AlLÉLY.

MEUNIER, Valérie, La société gallo-romaine à travers les traditions artistiques des stèles funéraires du Nord ouest de la Gaule du I $^{\text {r }}$ au III siècle, dir. E. BERTRAND.

\section{Histoire médiévale}

LEPROVOST, Céline, La pierre : matériau de construction et de réemploi dans les châteaux sarthois $d u X I^{e}$ au $X V^{e}$ siècle, dir. A. RENOUX.

Moga, Frédéric, La forêt de Sillé au Moyen Âge, dir. A. RENOUX. 
VoIsIn, Marie, L'Abbaye du Gué de Launay (XIT au XVe siècle), dir. A. RENOUX.

\section{Histoire moderne}

AlLARD, Marion d', Jacques de Beauvoir du Roure. Un gentilhomme protestant au XVII siècle, dir. D. Boisson.

DESNOYERS, Sandrine, Les mariages de Bonnétable 1673 à 1675 et de 1783 à 1785 , dir. L. BOURQUIN.

DuCHEMIN, Amélie, Les protestants d'Authon du Perche au XVIII siècle, dir. D. Bolsson.

GARREAU, Séverine, L'archet et le pinceau. Louis-Pierre Lhommeau, musicien et peintre au Mans à la fin du XVIII siècle, dir. S. GRANGER.

LANCELEUR, Angélo, Le château des évêques d'Yuré-L'Évêque - L'architecture au service du prestige social, dir. J.-M. CONSTANT.

PINHEIRO, Julia, Imprimeurs et libraires à La Flèche au temps des Jésuites. (1604-1762), dir. D. BoIsson.

POTIER, Emmanuel, Étude des idées d'un grand magistrat au temps des guerres de Religion : Claude Grouland, premier président du parlement de Normandie (1551-1607), dir. H. DAussY.

RaGo, Nicolas, La Société Royale d'Alençon (1762-1790), dir. L. BOURQUIN.

ROYER, Véronique, Le testament au Lude au XVIt siècle, dir. J.-M. CONSTANT.

\section{Histoire contemporaine}

Desile, Mélanie, Le Collège de Sillé-leGuillaume, dir. B. WACHÉ

DOMERGUE, Laure, Charles Dugasseau, Conservateur du Musée du Mans de 1856 à 1885, dir. F. LUCBERT, B. WACHÉ.

GAIGNARD, Michel, Jean Letourneau (1907-1986), député et ministre sarthois, dir. C. MANIGAND.

GAIGNARD, Sophie, Les mairies du canton de Sillé-le-Guillaume, dir. N. VIVIER.

GoRIN, Stéphane, La "bistrocratie " mancelle de l'entre-deux-guerres, dir. B. WaCHÉ.

Jamin, Sophie, L'émancipation de la femme à travers le veuvage à Sablé au XIXe siècle, dir. B. WACHÉ.

LAMBRET, Olivier, Léon Bourgeois et la société des Nations, dir. C. MANIGAND.
LEMAîTRE, Sophie, Naissance et évolution $d u 1^{e r}$ quotidien sarthois des origines (1868-1870) à l'affaire Dreyfus (1898), dir. C. MANIGAND.

LEMIERRE, François-David, L'Express et Jean-Jacques Servan Schreiber de 1969 à 1974, journaliste d'opinion et action politique, dir. C. MANIGAND.

ORAIN, Yannick, La politique sociale de la Caisse d'Épargne de Mamers 18701940, dir. N. VIVIER.

Ousman, Adam, Le sous développement de l'Afrique noire francophone à travers Ouest-France, dir. B. WACHÉ.

PAPIN-CRoIsÉ, Marie-Noëlle, Les réseaux de renseignement en Sarthe 19401944, dir. B. WACHÉ.

TeSTART, Jean-Benoît, Charles Gaudin de Saint-Rémy et Adolphe d'Espaulart : les relations des deux sarthois avec la vie artistique mancelle au XIXe siècle, dir. F. LUCBERT, B. WACHÉ.

\section{2}

\section{Histoire ancienne}

CACHAN, Miguel, La magie dans l'Antiquité romaine à travers l'œuvre d'Apulée, dir. M.-C. L'HUILLIER.

CHASLES, Erwan, Les femmes dans la société gallo-romaine. Étude des monuments funéraires, dir. E. BERTRAND.

EDON, Christelle, La place de l'enfant dans la peinture pompéienne, dir. E. BERTRAND.

LEMAîTRE, Marion, Les femmes dans la Rome mythique et archaïque, dir. A. ALLELY.

MARTIN, François, Les déclarations d'Hostis publicis dans la Rome républicaine, dir. A. AlLELY.

MEUNIER, Valérie, La société gallo-romaine à travers les traditions artistiques des stèles funéraires du Nord-Ouest de la Gaule du I r $^{\text {au }}$ III ${ }^{e}$ siècle, dir. E. BERTRAND.

\section{Histoire médiévale}

LEPROVOST, Céline, La pierre : matériau de construction et de réemploi dans les châteaux sarthois $d u \quad X l^{e}$ au $X V^{e}$ siècle, dir. A. RENOUX. 
Moga, Frédéric, La forêt de Sillé au Moyen Âge, dir. A. RENOuX.

VoIsIn, Marie, L'Abbaye du Gué de Launay (XII au XV siècle), dir. A. RENOUX.

\section{Histoire moderne}

BRIÈRE, Sandrine, Les enfants trouvés dans la généralité d'Alençon à la fin du XVIII siècle, dir. F. PITou.

CALIBRE, Annie, L'hôtel Le Prince ou la matérialisation de la notabilité urbaine, dir. J. M. CONSTANT.

CoQuIN, Ludovic, Regard sur l'habitat paysan du Bas Maine au XVII' siècle. L'exemple de la région de Chailland, dir. F. PITou.

DELLIÈRE, Laëtitia, Les filles mères dans le Haut-Anjou et le Bas-Maine au XVIII ${ }^{e}$ siècle, dir. F. PITOU.

GENEAU, Catherine, Unions percheronnes : les mariages, dir. S. GRANGER.

LENORMAND, Dominique, Le nouveau grenier à sel de Craon, dir. F. PITOU.

LEVALET, Manuela, L'habitat des bourgs et des campagnes en Champagne mancelle à la fin $d u$ xVIII siècle, dir. F. PITOU.

LHOPITAL, Claire-Séverine, Une famille dans la tourmente révolutionnaire : les Bastard de Fontenay, dir. J.-M. CONSTANT.

MonNIETTE, Sébastien, Les protestants d'Athis et de ses environs sous le règne de Louis XIV (1643-1715), dir. D. BOISSON.

Moron, Céline, Les Renaissances du château de Malicorne (Sarthe), dir. J.-M. CONSTANT.

OudILL-LEROY, Virginie, Les marchands Loupéens de 1694 à 1719, dir. J.-M. CONSTANT.

Poisson, Guillaume, Regard d'un voyageur français sur la Suisse au siècle des Lumières, dir. J.-M. CONSTANT.

SuZANNE, Jean-François, La vie municipale à Mortagne-au-Perche (17661790), dir. L. BOURQUIN.

Trifault, Cécile, Gros plan sur le bâti rural dans la région de Bonnétable, dir. J.-M. CONSTANT)

VoullLemy, Adeline, Les idées politiques paysannes dans le Saosnois en 1789, dir. J.-M. CONSTANT.

\section{Histoire contemporaine}

ANFRAY, Maggy, L'émancipation des femmes dans la Sarthe - années 20, dir. N. VIVIER.

BACHELOT, Bruno, La mise en place de la législation anticommuniste à la SNCF 1939-1942, dir. N. VIVIER.

BLONDEL, Nausicaa, L'art normand dans la tourmente 1940-1944, dir. B. WACHÉ.

BREGER, Gaëlle, L'évolution du cadre matériel et agricole des paysans des Coëvrons au XIX siècle (1830-1890), dir. B. WACHÉ.

CôME, Jérôme, Le théâtre de Saint-Calais (1842-1846), dir. B. WACHÉ.

DELATTRE, Maxime, L'épuration administrative à la SNCF à la Libération, dir. N. VIVIER.

DuTHEIL, Alexandra, La construction des casernes au Mans après 1870, dir. N. VIVIER.

FouCAULT, Caroline, La mise en valeur du patrimoine dans le canton de Nocé, dir. N. VIVIER.

GAUDRÉ, Benjamin, Place et interprétations de la commune de Pons dans les manuels scolaires d'histoire (18812000), dir. B. WACHÉ.

GHALEB, Mohamed, L'évolution économique et sociale de la CFS de 1929 à 1961, dir. N. VIVIER.

GUIBERT, Fabien, Le détachement de main-d'œuvre de la SNCF aux chemins de fer allemands 1842-1844, dir. N. VIVIER.

JOURDREN, Jeanne, Les Fêtes des reines de Cornouailles 1923-1948, contributions des fêtes traditionnelles au maintien de l'identité régionale en Bretagne, dir. B. WACHÉ.

LE Bossé, Antony, Les ouvriers de la Sarthe sous le Second Empire, dir. N. VIVIER.

MAILY, David, La construction des mairies au XIXe siècle dans trois communes : Ballon, La Bazoge, Monbizot, dir. N. VIVIER.

PAgeot, Alexandra, La contribution des femmes de la Sarthe à la Résistance, dir. N. VIVIER.

Patault, Annabelle, La doctrine nazie vue par quatre journaux canadiens français 1934-1939, dir. B. WACHÉ. 
Porcher, Aurélie, Les potiers de Malicorne au XIXe siècle, dir. N. VIVIER.

Rigollet, Laurent, Les blessés de la Grande Guerre dans la Sarthe, dir. C. MANIGAND.

SAISON, Laure, Les événements de 1968 au Mans. Le mouvement étudiant, dir. B. WaCHÉ.

VALLÉE, Delphine, L'industrie nourricière dans le département de la Sarthe au XIX siècle, dir. N. VIVIER.

\section{1}

\section{Histoire ancienne}

BOURROUX, Antoine, La guerre novatrice chez les Achéménides et les Argéades, dir. A. TOURRAIX.

Chauvin, Jean-Pierre, Le musée municipal d'Ernée. Étude du fonds galloromain, dir. E. BERTRAND.

CORMIER, Benjamin, Puissance romaine et diplomatie : les témoignages d'Ammien Marcellin et de Julien, dir. M.-C. L'HUILLIER.

FLANDRIN, Solenn, La féminité chez Julien, dir. M.-C. L'HuilLIER.

FRÉMONT, Christophe de, Athènes et la Thrace du vI au IV siècle av. J.-C., dir. A. TOURRAIX.

GRANGER, Aurore, Les divinités de la Gaule de l'Ouest, dir. A. AlLELY.

JAOUEN, Caroline, Les bibliothèques grecques hellénistiques : personnel et lectorat, dir. A. TOURRAIX.

LOISEAU, Christophe, Métal et construction du grand temple de la Forêterie (Allonnes, Sarthe), dir. E. BERTRAND.

MARCHAND, Aurélie, Représentations des femmes dans l'Histoire d'Auguste, dir. M.-C. L'HUILLIER.

PICARD, Alexandra, Les femmes de la nobilitas de la fin de la République au début du Principat, dir. A. AlLELY.

WAWRZYNIAK, Grégory, Les acteurs au $v^{e}$ siècle av. J.-C. en Grèce Ancienne, dir. B. LE GUEN.

\section{Histoire médiévale}

AlBERT, Céline, La pierre dans la construction des églises sarthoises (IX'-XV siècle), dir. A. RENOUX.
BourgoIn, Stéphane, Mottes et maisons fortes dans le canton de Sablé-surSarthe au Moyen Âge, dir. A. RENoux.

Chavigny, Thiphaine de, La Faigne, Seigneurie puis châtellerie du XII $a u$ $X V I^{e}$ siècle, dir. A. RENOUX.

DOUMERC, François, L'aristocratie $d u$ Maine au haut Moyen Âge. (fin Ve-fin IX siècle, dir. A. RENOUX.

LE CLOëREC, Ludovic, Église et aristocratie dans les confins du Maine avec la Bretagne ( $V^{e}-X I^{e}$ siècle), dir. A. RENOUX. MÉmin, Xavier, Les églises et les paroisses du doyenné de la Quinte du Mans au Moyen Âge, dir. A. RENOUX.

\section{Histoire moderne}

COTTERET, Betty, Les chirurgiens de La Flèche au XVIII ${ }^{e}$ siècle, dir. L. BouRQuIN.

Deligne, Thomas, La population d'Yvrél'Évêque de 1760 à 1792. Étude de quelques aspects démographiques, dir. L. BOURQuin.

LANDAIS, David, Les Hôpitaux du Lude : de leurs fondations à la Révolution, dir. L. BOURQUIN.

LEBossé, Corine, La réforme d'un hôpital du Maine au XVIII siècle : l'exemple de Montfort-le-Rotrou et Pont-de-Gennes (1705-1720), dir. L. BOURQUIN.

MADELAINE, Vanessa, Les contrats de mariage à Assé-Le-Riboul de 1785 à 1795, dir. L. BOURQuIN.

MARIE, Magaly, Le canton de Bonnétable sous le Directoire, dir. J.-M. Constant.

MAYNIER, Nicolas, L'Assistance publique au Mans pendant la Révolution, dir. J.M. CONSTANT.

TACHEAU, Vincent, Étude matérielle et sociale de la vie de trois marchands de textile Féchois à la fin du XVIII siècle (1770-1795), dir. L. BouRQuIN.

TARON, Arnaud, La formation d'une bande armée au Lude : opposition à l'autorité royale ou acte de brigandage sous la seconde restauration, dir. F. Pitou.

VilletTE, Jérôme, La Cité mancelle des marchands drapiers et merciers sous l'Ancien Régime, dir. L. BOURQuiN. 


\section{Histoire contemporaine}

AdEN Mahamoud, Fathia, La présence française en Côte française des Somalies (1936-1960), dir. C. MetzGER.

BordiER, Julien, Georges Bernanos, témoin des années 1930 (1932-1938), dir. B. WACHÉ.

Bouland, Magaly, Les Bouchers du Mans au XIX siècle, dir. N. VIVIER.

BRAUX, Mélanie, L'occupation allemande au Mans en 1871, dir. N. VIVIER.

BuREL, Florent, La présence des militaires américains en Sarthe 19171919, dir. N. VIVIER.

CARRÉ, Bertrand, Évolution des locomotives diesel de ligne à la SNCF 1950 1975, dir. N. VIVIER.

CARRIOT, Magalie, Le comice de Châteaudu-Loir 1839-1914, dir. N. VIVIER.

COLLET, Joakim, Les femmes en Sarthe et en Mayenne à l'heure de la guerre civile 1713-1796, dir. B. WACHÉ.

CouAnON, Carole, Les femmes dans le monde rural dans la Sarthe (18481870), dir. N. VIVIER.

DELOZIER, Linda, Les femmes dans le canton d'Alençon 1880-1900, dir. N. VIVIER.

DereszowsKI, Céline, La chapelle de la Providence à La Flèche (1845-1864), dir. B. WACHÉ.

DESNOS, Sylvain, L'occupation prussienne de la Sarthe en 1815, dir. N. VIVIER.

DeulofEu, Nicolas, Foires et Marchés du Mans au XIX $x^{e}$ siècle, dir. N. VIVIER.

DROPSY, Sophie, La sage-femme : son rôle et sa vie entre la fin de la Révolution et le milieu du XIX siècle. dir. B. WaCHÉ.

DuPONT, Virginie, Le ravitaillement alimentaire en Sarthe après la Libération, dir. B. WACHÉ.

EL BAHLOUL, Bahija, La sorcellerie dans le Maine $X I X^{e}-X X^{e}$ siècle, dir. P. FoucAult et N. VIVIER.

ESNEAULT, Matthias, La guerre aérienne en Normandie 1940-1945, dir. N. VIVIER.

FERnANDES, Paulo, La Caisse d'Épargne de Mamers 1836-1872, dir. N. VIVIER.

FonTAINE, Anne-Cécile, Les notables du canton de Montmirail 1815-1848, dir. N. VIVIER.
GuILlET, Franck, L'aspect des Frères de l'Instruction chrétienne dans les colonies françaises : Guadeloupe, Martine et Guyane de 1836 à 1860, dir. B. WACHÉ.

HAUDRY, Nelly, La noblesse autour du Grand Lucé du début du XVIII siècle à la veille de la Révolution, dir. L. BOURQuin et J.-M. CONSTANT.

HATTON, Valérie, Les bombardements au Mans pendant la $2^{e}$ guerre mondiale, dir. B. WACHÉ.

LANCELEUR, Michaël, Les rentes viagères, dans la Sarthe 1875-1898, dir. N. VIVIER.

MANTEY, Bénédicte, L'organisation de vie et de travail des religieuses hospitalières de Saint-Joseph au sein de l'Hôtel-Dieu de Montréal, dir. B. WACHÉ. MORÈRE, Joël, Les aides alliées à la Marine française durant la Seconde Guerre mondiale, dir. C. METZER.

NiCOLAS, Émilie, L'Action française dans la Sarthe jusqu'en 1926, dir. B. WACHÉ.

PALlaVicini, Jérôme, La réception d'Ernest Renan dans la Sarthe, dir. B. WACHÉ.

THOMAIN, Cédric, Les relations francoallemandes vue par la presse sarthoise, (1923-1924), dir. C. MEtzGer.

Tolmont, Solène, L'infanticide en Sarthe (1825-1830), dir. N. VIVIER.

\section{0}

\section{Histoire ancienne}

BILY, Gwénola, L'aristocratie gallo-romaine au III IV $^{e}$ et $V^{e}$ siècle d'après les sources littéraires, dir. M.-C. L'HuILLIER.

DELPEUX, Virginie, L'abandon des enfants à Rome : des origines aux Antonins, dir. A. AlLELY.

DuFEU, Laurent, Les disciples des Sophistes en Grèce classique, dir. A. TOURRAIX.

Foulon, Thierry, La région des Détroits entre Grecs et Perses, dir. A. TOURRAIX.

JEAN-MARIE, Lynda, La vision des chrétiens sur les cultes égyptiens aux III et IV siècles, dir. M.-C. L'HuILLIER.

JeuneHomme, Frédéric, Le Maître de Cavalerie à Rome durant la République, dir. A. Allely. 
KÜHNER, Valérie, Les cultes d'Athéna en dehors d'Athènes, dir. A. TOURRAIX.

LASSAY, Céline, Omina imperii Présages et propagande sous les JulioClaudiens, dir. E. BERTRAND.

POIRIER, Elsa, La perception de l'histoire $d u I^{e}$ siècle au travers des représentations spatiales et paysagères d'Ammien Marcellin, dir. M.-C. L'HUILLIER.

\section{Histoire médiévale}

BELLENCONTRE, Hervé, La conquête du pouvoir dans le Maine par le bâtard normand (1048-1087), dir. A. RENOUX.

BOUVIER, Ludwig, Les sermons joyeux à la fin du Moyen Âge, dir. A. Renoux.

CATHERINE, Olivier, Les logis abbatiaux des abbayes bénédictines et cisterciennes dans le diocèse de Bayeux à la fin du Moyen Âge, dir. A. RENOUX.

Destable, Erwan, La Seigneurie de la Chartre sur le Loir au Moyen Âge, dir. A. RENOUX.

LEGUAY, Sophie, Mottes et hébergements fossoyés dans le canton de Bouloire (XIe-XVe siècle), dir. A. RENOUX.

PERROT, Nicolas, La baronnie de la Milesse à la fin du Moyen Âge, dir. A. RENOUX.

Porteneuve, Véronique, Mottes et maisons fortes dans le canton de StPaterne (XI -XVe siècle), dir. A. RENOUX.

\section{Histoire moderne}

BARBIER, Élise, L'église de Villaines-sousMalicorne, patrimoine architectural et artistique, dir. A. DUPRAT.

BARDET, Jimmy, Les droits seigneuriaux dans la châtellerie de Grandchamp, dir. L. BOURQuIN.

BEDOUIN, Olivier, Le couvent des minimes de Sillé-le-Guillaume. Une communauté conventuelle sur une terre du HautMaine au XVIf siècle, dir. L. BoURQUIN.

BERVAS, Laurent, Ascension et disparition d'une famille aux XVII et XVIII siècles dans le Haut Maine : les Jeudon de Segrais, dir. L. BourQuin.

Bouvet, Valérie, Joseph-Jean Portier (1751-1831), Marchand-cartier-dominotier-imagier-papetier en la ville du Mans, dir. A. DUPRAT.
COLLET, Cédric, Naître, convoler, mourir. Étude du registre de baptêmes, mariages, sépultures à Sargé-lès-LeMans (1764-1792), dir. L. BouRQUIN.

Coupé, Cyril, L'ancien hôpital SaintJoseph de La Flèche aux XVII et XVIII siècles, dir. J.-M. Constant.

FAILLEAU, Gaëlle, L'infanticide au travers de huit procès (1702-1768) dans le Haut Maine, dir. J.-M. CONSTANT.

ForTIER, Jérôme, La baronnie de Touvoie au XVIII siècle, dir. L. BOURQUIN.

FrançoIs, Sébastien, Le Grand Lucé dans le tumulte révolutionnaire 1795-1797, dir. A. DuPRAT.

GAUCHER, Aurélia, Pierre-François Godard (1768-1838) graveur à Alençon, dir. A. DuPRAT.

GERMOND, Stéphanie, Entre Beauce et Perche : l'architecture des fermes dans la baronnie de Courville (1740-1830), dir. J.-M. CONSTANT.

HEINTZ-GRESSER, Lise-Marie, Les maisons de tisserands à Fresnay-sur-Sarthe(18201870), dir. J.-M. CONSTANT.

JULIEN, Alexandre, Le délit de chasse dans le Maine au XVIII siècle, dir. L. BOURQUIN.

LECONTE, Charlotte, Jacques-Rigomes Bazin (1771-1818). Itinéraire d'un révolutionnaire démocrate, dir. A. DUPRAT.

LIGNEUL, Jérôme, Médecins et maladies dans la Généralité d'Alençon (17371771), dir. J.-M. CONSTANT.

NAVAR, Julien, Les métairies du Saosnois au XVIII siècle, dir. L. BouRQuIN.

PERROTEL-GuitTET, Sabrina, L'Instruction élémentaire dans la Région de SaintCalais : des petites écoles d'Ancien régime aux écoles de la République, dir. J.-M. CONSTANT.

RENAULT, Nicolas, Les conditions de vie des prêtres du Bas Maine et du Haut Anjou au XVIII siècle, dir. J.-M. CONSTANT.

Rousseau, Laurence, Portes marinières et navigation sur la Mayenne au XVIII siècle, dir. J.-M. CONSTANT.

Souvré, Arnaud, L'abbaye Notre-Dame des Clairets au XVIII siècle : une clé de femmes cisterciennes dans le Perche, dir. J.-M. CONSTANT. 
TESBER, Andréa, L'architecture de la bande dessinée, dir. A. DuPRAT.

Termeau, Thierry, La réforme de l'abbaye Saint-Pierre-de-la-Couture par la congrégation de Saint-Maur (16561661), dir. L. BOURQUIN.

\section{Histoire contemporaine}

ABDOURAHMAN MAHOMOUD, Fatouma, La côte française des Somalies (18821945), dir. C. METZGER.

AuvraY, Miguël, La communauté européenne de défense vue à travers la presse française (Le Monde, L'Humanité, Ouest-France, Le Maine Libre, La Dépêche Libre du Maine), dir. C. MetzGer.

BERBEL, Diego, L'Europe Jazz Festival et la politique culturelle de la ville du Mans (1977-2000), dir. B. WACHÉ.

BEUVIER, Maud, Le camp d'internement de Précigné, dir. B. Waché.

BLAISE-MARTIN, Hélène, Le canton d'Alençon face à la politique religieuse révolutionnaire (1790-1799), dir. B. WACHÉ.

BORDIER, Christophe, Le comice agricole de Nogent-le-Rotrou, dir. N. VIVIER.

BuLlot, Ludovic, Les relations entre la France et le Maroc de 1957 à 1961 à travers les documents diplomatiques français, dir. C. METZGER.

Chevallier, Magali, Étude d'une scierie de l'Orne, dir. N. VIVIER.

Duval, Angélique, La retombée des lois anti-congréganistes de 1880 à 1906 en Mayenne : exemple de la congrégation de la charité de Notre-Dame d'Évron, dir. B. WaCHÉ.

ETIEMBLE, Gaël, La vie des gardes forestiers dans l'inspection d'Alençon 18751925, dir. N. VIVIER.

FouCAULT-DAVAL, Colette, Les catholiques et l'art religieux 1919-1939, dir. B. WACHÉ.

HuAulmÉ, Christophe, La vie des ardoisiers de Renazé 1894-1939, dir. N. VIVIER.

JouBIN, Frédéric, La censure de la presse pendant la première guerre mondiale, dir. C. MeTZGER.

LAPULY, Arnaud, Le travail des enfants dans la Mayenne au XIX siècle, dir. N. VIVIER.
LAUNAY, Vincent, Le comice agricole du Mans, dir. N. VIVIER.

MilCENT, Nathalie, La congrégation de la Miséricorde de Sées, dir. B. Waché.

MiLET, Guillaume, Laval dans la grande guerre, une société de l'arrière, dir. B. WaCHÉ.

NEZAN, Sandrine, Le culte marial dans le Nord-Mayenne durant la seconde moitié du XIXe siècle (1850-1900), dir. B. WACHÉ.

NICOLLE, David, Le corps expéditionnaire français en Indochine de décembre 1946 à octobre 1954 : l'exemple des Anciens combattants sarthois, dir. C. METZGER.

OURIAD, Fatima, Les sages-femmes dans la Sarthe au XIX siècle, dir. N. VIVIER.

PIQUET, Edwige, Les étrangers de nationalité allemande en Sarthe du début du XIX siècle à 1870, dir. C. MetzGER.

PluT, Gilles, Les Anciens combattants d'Algérie : 40 ans après à travers leurs témoignages, les archives militaires et les sondages de presse, dir. C. MetzGer.

PoIsson, Ludivine, L'enseignement primaire des filles par la congrégation des sœurs de la charité d'Evron en Mayenne au XIX (1803-1880), dir. B. WACHÉ)

Provost, Mélanie, Le planning familial dans la Sarthe, dir. N. VIVIER.

REMARS, Céline, Les étrangers en Sarthe à l'heure de la Grande Guerre, dir. B. WACHÉ.

Rousseau, Sandy, Étude comparée des comices du Lude et de Conlie 18381859, dir. N. VIVIER.

SANSÉAU, Hélène, L'Ouest-Éclair dans la Grande Guerre - Un quotidien régional face au devoir d'information, dir. B. WaCHÉ.

THOMAS, Jean-Noël, L'établissement de l'administration préfectorale dans le département de la Sarthe sous le Consulat (1800-1804), dir. B. WACHÉ.

TRÉOL, Mickaël, Monographie de l'usine de Bourberaye 1792-1863, dir. N. VIVIER.

Yvard, David, Le Sud Vietnam de juillet 1954 à mai 1957. Le passage de relais entre la France et les États-Unis 
à travers les Documents diplomatiques français, dir. C. METZGER.

\section{9}

\section{Histoire Médiévale}

BouEME, Karine, Les dédicaces d'églises dans le Haut Maine au Moyen Âge, dir. A. RENOUX.

CHEVALLIER, Jan, Les édifices religieux dans la partie sud de l'archidiocèse de Blois ( $X^{e}-X V^{e}$ siècle), dir. A. RENOUX.

Decosse, Anne, La topographie socioéconomique du Mans à la fin $d u$ Moyen Âge, dir. A. RENOUX.

DuBin, Laurence, Les églises du doyenné de Beaumont-sur-Sarthe au Moyen Âge, dir. A. Renoux.

MARQUET, Valérie, Inventaire des mottes et maisons fortes dans le canton de Montfort-le-Gesnois ( $X^{e}-X V^{e}$ siècle), dir. A. RENOUX.

Plantin, Sylvère, Églises et paroisses du doyenné de La Ferté-Bernard au Moyen Âge, dir. A. RENoux.

Roux, Christine, Mottes et hébergements dans le canton de Tuffé (Xle-XVe siècle), dir. A. RENOUX.

VAlLoIS, Claire, La seigneurie de Bazouges-sur-le-Loir (XIVe-XVI siècle), dir. A. RENOUX)

Vetillard, Sébastien, Notaire, poète et historien - Guillaume le Doyen témoin de son temps (fin $X V^{e}$-début $X V^{e}$ siècle), dir. A. RENOUX.

\section{Histoire moderne}

Bignon, Yann, Principe égalitaire des successions roturières dans le Maine au XVIII siècle, dir. A. FILLON.

Boulay, Sandrine, Les couteliers du Mans au XVIII siècle, dir. A. FILLON.

CADIEU, Stéphanie, Les marchands hôtes de Malicorne de 1750 à 1780, dir. A. FILLON.

CARETTE, Marie, les idées politiques paysannes dans le district d'Amboise en 1789 (vallée du Cher), dir. J.M. CONSTANT.

CouIn, Céline, Le problème des épidémies et maladies épidémiques dans la généralité d'Alençon (1776-1786) d'après les mémoires de médecine envoyés à la Faculté Royale de Médecine, dir. A. Fillon.

DAVOUDET, Virginie, Les orfèvres du Mans au XVIII siècle, dir. A. FILLON.

FLEURY, Catherine, Ecommoy sous la Révolution de la Ire République 17891799, dir. J.-M. CONSTANT.

FOUREY, Céline, Les villes après la réforme dans le Duché de Wintemberg (1534-1567), dir. J.-M. CONSTANT.

GuÉRIN, Maud, La chartreuse du Val Dieu dans la $2^{e}$ moitié du XVIII siècle, dir. J.M. CONSTANT.

GuY, Graziella, La vie municipale à Montmirail (1789-1799), dir. J.-M. CONSTANT.

JugEn Sarah, Le Prieuré de Champagné au XVIII siècle (1700-1799), dir. J.-M. CONSTANT.

LEVEAU, Laure, Les idées politiques paysannes dans le district d'Amboise en 1789 (vallée de la Loire), dir. J.-M. CONSTANT.

\section{Histoire contemporaine}

AsSIER, Denis, Les sapeurs-pompiers de l'Orne au XIX siècle, dir. N. VIVIER.

BEAUdouin, Stéphane, La bibliothèque municipale du Mans d'une guerre à l'autre, 1914-1945, dir. B. WACHÉ.

BuIn, Thibault, L'assasinat politique d'Yitzchak Rabin; les différentes visions de la presse écrite et de la presse orale sur l'événement, dir. N. VIVIER.

Champ, Adeline, Les Marraines de guerre (Première et Seconde Guerre mondiale), dir. N. VIVIER.

Chevreul, Céline, Marie, un cour simple. Un témoignage d'une pratique religieuse intense au cour de la campagne mayennaise, dir. B. WACHÉ.

CORBEAU, Stéphanie, L'enseignement agricole dans la Mayenne 1835-1935, dir. N. VIVIER.

Dosso, Julia, Les Italiens en Maine-etLoire 1860-1920, dir. B. WaCHÉ.

FOUQUERAY, David, Dom Piolin, un moine de Solesmes, un historien de l'Église du Maine, dir. B. WACHÉ.

GABILLET, Fabien, Les échos de la séparation de l'Église et de l'État en France dans 
la presse canadienne française : reflet d'une société en mutation?, dir. B. WACHÉ.

HERVÉ, Christine, L'affaire Dreyfus vue par la presse catholique sarthoise - La Croix du Maine 1894-1906, dir. B. WACHÉ.

Josso, Anthony, Sensibilité des anciens combattants de la Sarthe, à travers les journaux de l'après-guerre 1918-1928, dir. N. VIVIER.

LAANAYA, Saouad, Le théâtre et le cinéma au Mans de 1914 à 1929, dir. B. WACHÉ

LANGLET, Aude-Marie, L'Ouest-Éclair et le national socialisme, janvier 1923janvier 1933, dir. B. WaCHÉ.

LAUNAY, Arnault, La maçonnerie française dans les années 1930 à travers l'exemple d'une loge sarthoise : Les Amis du Progrès, dir. B. Waché.

Le Bideau, Samuel, Délits d'opinion et répression politique dans l'Orne sous la Restauration, dir. B. WACHÉ.

LECONTE, Nathalie, La vie culturelle dans le Cotentin 1936-1947, dir. B. WACHÉ.

LEROUX, Loïc, L'âge d'or des tramways électriques au Mans 1897-1914, dir. B. WACHÉ.

LESASSIER, Céline, Regards sur la création de l'entre-deux-guerres : théâtre, littérature et cinéma en France 1919-1939, dir. B. WACHÉ.

LEVEAU, Gaëlle, L'exposition de 1911 en histoire et en image, dir. A. DUPRAT et B. WACHÉ.

LOCHET, Magalie, L'abbé Lochet, vicaire à Notre-Dame de la Couture au Mans, dir. B. WACHÉ.

MAuBoussin, Virginie, L'aménagement de la place de la République au Mans dans la seconde moitié du $X X^{e}$ siècle, dir. B. WACHÉ.

MoAl, Emmanuelle, L'ouvre de l'Automobile Club de l'Ouest pendant la Grande Guerre, dir. B. WACHÉ.

MOREAU, Aurélien, Intellectuels révolutionnaires en guerre d'Algérie : "socialisme ou barbarie ", dir. B. WACHÉ.

MourahiB-BIDAR, Khadija, L'abbé Baret, prêtre intellectuel manceau, dir B. WaCHÉ

PEREIRA, Anne-Zita, L'Église et le cinéma des années soixante : censure ou promotion?, dir. B. WACHÉ.
PERRIÈRE, Joran, L'instruction primaire dans l'arrondissement du Mans : l'exemple de La Bazoge, dir. B. WACHÉ. RIVERON, Patrice, La peste bovine en Mayenne - 1871, dir. N. VIVIER.

RIvET, Nicolas, Deux églises dans un siècle en mutation : Notre-Dame de Cholet et Montligeon au XIX siècle, dir. B. WACHÉ.

Sauvagnac, Dorothée, Missions et missionnaires en Sarthe à travers l'exemple de Paul-Auguste Blanchet (1852-1912), dir. B. WaCHÉ.

VIRLOUVET, Stéphanie, Le comice agricole de la Ferté-Bernard - XIX siècle, dir. N. VIVIER.

\section{8}

\section{Histoire ancienne}

BEURET, Brice, L'image des Germains à travers les oeuvres d'Ammien Marcellin et Jordanès, dir. J. BIARNE.

Buin, Stéphanie, Le culte des Muses en Grèce ancienne, A. TOURRAIX.

DELION, Virginie, La figure de l'Empereur Constantin à travers les historiens ecclésiastiques (IVe-ve siècles, dir. J. BIARNE.

Dorizon, Karine, Le vêtement féminin et enfantin dans le monde romain aux II et III siècles, dir. J. BIARNE.

DuBraY, Virginie, Les statues à Rome d'après Pline l'Ancien, dir. J. BIARNE et C. BUSTANY.

GAIGNON, Maryse, Les représentations iconographiques de divinités guerrières à Rome du $\mathrm{I}^{\mathrm{er}}$ siècle avant J.-C. jusqu'à Auguste, dir. J. BIARNE et C. BUSTANY.

GALMARD, Sandrine, Le temporel de l'évêché du Mans, à travers les Actus, de Julien à Hadoind, dir. J. BIARNE.

HARAN, Valérie, Le terroir de la Sarthe aux époques gallo-romaine et franque, dir. J. BIARNE.

LEMONNIER, Nicolas, L'Empereur et la Cité, mise en scène du pouvoir et imaginaire collectif dans l'Antiquité tardive d'après les Panégyriques gaulois, dir. J. BIARNE.

Normand, Jérôme, L'île de Chios à l'époque classique, dir. P. BRUN. 
RobBe, Pascal, L'île de Naxos à l'époque archaïque, dir. P. BRUN.

SAULNIER, Benoît, La cité de Samothrace, dir. P. BRUN.

\section{Histoire Médiévale}

ALATERRE, Christophe, Le rocher d'Entrammes, village de potiers de la fin du Moyen Âge au XVIII siècle, dir. A. RENOUX. Chatelain, Hélène, Étude de l'inventaire du mobilier de Charles $V$, roi de France (1379-1380), dir. A. RENOUX.

CHIRAT, Franck, Mottes et maisons fortes dans le canton de Marolles-les-Braults ( $X^{e}-X V^{e}$ siècle), dir. A. RENOUX)

DESCAMPS, Natacha, La baronnie de La Flèche à la fin du Moyen Âge, dir. A. RENOUX.

ERNOUX, Ludovic, La forêt de Perseigne à l'époque médiévale, dir. A. Renoux.

FouILLET, Thierry, Le chapitre cathédral du Mans et ses chanoines (13831448), dir. A. RENOUX.

GARRAULT, Johann, L'enseignement de l'histoire médiévale du XIII ${ }^{e}$ au XVe siècle en France de 1870 à 1939, dir. A. RENOUX.

GASCHET, Valérie, La châtellerie de Saint Calais à la fin du Moyen Âge, dir. A. RENOUX.

GÉRARD, Sylvain, Le château de Ballon et ses seigneurs à la fin du Moyen Âge, dir. A. RENOUX.

GoussET, Jean-Michel, Les fortifications urbaines de Laval du XI au XVIII siècle, dir. A. RENOUX.

HARDY, Julien, Les chapelles castrales et manoriales du Maine. Inventaire pour l'arrondissement de Mamers, dir. A. RENOUX.

HUMILIER, Stéphane, La seigneurie de Courville à la fin du Moyen Âge, dir. A. RenOuX.

LACOUE, Isabelle, Les églises rurales $d u$ doyenné de Sillé-le-Guillaume à l'époque médiévale, dir. A. RENoux.

LE BIHAN, Frédéric, La chevalerie du XII au $X V^{e}$ siècle dans le cinéma français et anglais d'après guerre, dir. A. RENOUX.

LEFEBVRE, Stéphanie, Mottes et maisons fortes du canton de Bouloire (XI-XVI siècle), dir. A. RENOUX.
MASNIÈRE-BurEAu, Isabelle, La ville de $L a$ Ferté-Bernard au XIVe et $X V^{e}$ siècle, dir. A. RENOUX.

Oudin, Frédéric, La seigneurie de Poncésur-le-Loir du XI au XV siècle, dir. A. RENOUX.

PIRON, Céline, La sculpture romane du XIe siècle au Mans : étude des chapiteaux du chœur à l'église de La Couture, dir. A. RENOUX.

QuAIREAU, Sandrine, Sainte Suzanne, son château et son bourg à la fin du Moyen Âge, dir. A. Renoux.

RAMOND, Jean-Michel, La collégiale Notre-Dame de Sillé-le-Guillaume à la fin du Moyen Âge, dir. A. RENoux.

\section{Histoire moderne}

AUDIBERT, David, Épiciers du Mans au XVIII siècle, dir. A. FILLON.

BESSIN, Gaëlle, Une seigneurie au nord du Haut Maine au XVII siècle : visions patrimoniales et sociales du Vicomte de Meujot de Champfleur, dir. J.-M. CONSTANT.

BouvET, Élodie, Les artisans de la Suze-surSarthe dans la $2^{e}$ moitié du XVIII siècle, dir. A. FILLON.

COUEllier, Mélanie, La place de la République du Mans : aménagement et statuaire de la Révolution à la fin du XIXe siècle, dir. A. DUPRAT.

Cousin, Charles-Emmanuel, La société de Château-l'Hermitage à la veille de la Révolution, dir. A. FiLlon.

DelaPERrElle, Caroline, Cloches et fondeurs de cloches de la région du Mans du XVI au XVIII siècle, dir. A. FILLON.

DeVReKer, Sabrina, Les bouchers du Mans au XVIII siècle, dir. A. FILLON.

Euckmans, Natacha, Densité nobiliaire et noblesse dans le doyenné de Lignières la Carelle du XVI au XVIII siècle, dir. J.M. CONSTANT.

GAIGNARD, Sandrine, Les idées politiques paysannes du district de Luynes-enTouraine en 1789 à travers les doléances, dir. J.-M. CONSTANT.

Gaignon, Gaël, Les biens de Châteaul'Hermitage au XVII' siècle, dir. A. FILLON.

Garreau, Rénald, Louis Revelière. Parcours d'un notable 1790-1830, dir. A. DUPRAT. 
GOUFFIER, Céline, Les idées politiques paysannes dans le district de Montbazon en Touraine en 1789, dir. J.-M. CONSTANT.

GUERNEvE, Sonia, Les cordonniers du Mans au XVIII siècle, dir. A. FILLON.

HAYER, Yohann, La fabrique de Mézieres sur-Ponthouin au XVIII ${ }^{e}$ siècle, dir. A. FILLON.

HINAULT, Sandrine, La seigneurie d'Argentelles ou la place d'un cadet dans la société nobiliaire $d u$ xиII $e^{e}$ siècle, dir. J.M. CONSTANT.

LAMBALle, Laure, Pâtissiers manceaux au XVIII ${ }^{e}$ siècle, dir. A. FILLON.

LEPOITEVIN, Nicolas, Les idées politiques paysannes d'après les cahiers de doléances de 1789, dans le sud-est du baillage de Tours (vallée dite Creuse de la Claie Pays de Brenne), dir. J.-M. CONSTANT.

MAILLARD, Céline, L'image de la résistance intérieure française au temps de la Seconde Guerre mondiale à travers quelques films français 1945-1996, dir. A. DUPRAT.

MARChAND, Sandra, Les idées politiques paysannes d'après les cahiers de doléances de 1789 dans l'ouest du baillage de Mortagne au Perche, dir. J.-M. CONSTANT.

MARIAUX, Yann, La découverte de l'Amérique vue par les manuels scolaires (1907-1997), dir. A. DuPRAT.

MARIE, Rachel, Charles Douglas, J. du Ronceray. Physiocrate, dir. J.-M. Constant.

PAgeot, Corinne, Le vêtement populaire rural de 1729 à 1779 en Anjou, dir. A. FILLON.

PhILIPPE, Sébastien, Les moulins de la paroisse de Gourdaine et le mode de vie des meuniers dans la $2^{e}$ moitié du XVIII siècle, dir. A. FILLON.

PICHARD, Pierre, Roi, noblesse, Parlement et peuple : séditions et réconciliations - imagerie politique des guerres de religion (1559-1594), dir. A. DUPRAT.

РОтЕ́, Julia, Les idées politiques paysannes dans le baillage d'Alençon (entre la haute vallée de la Sarthe et celle de l'Orne en 1789), dir. J.-M. CONSTANT.

Rigaud, Sophie, Les sociétés de LibrePensée dans la Sarthe (1880-1905), dir. B. WACHÉ.
SALMON, David, La noblesse dans la baronnie normande du Mêle-sur-Sarthe aux XVII et XVII siècles, dir. J.-M. CONSTANT.

Simao Ramalho, Claudia, Le marquisat de la Varenne à La Flèche au XVIII siècle, dir. J.-M. CONSTANT.

TAINTURE, Yannick, Les idées politiques paysannes en 1789 dans la vallée de la Risle : étude quantitative des doléances, dir. J.-M. Constant.

TRESSERAS, Magali, Le théâtre révolutionnaire à travers les mémoires d'un soldat de la République, dir. A. DUPRAT.

Uzu, Véronique, Louis Basse, un notable manceau, dir. M. AuFFRET et B. WACHÉ.

Vankersschaever, David, Cossé-le-Vivien et ses environs à travers les métiers dans la $2^{e}$ moitié du XVIII siècle, dir. A. FILLON.

\section{Histoire contemporaine}

AUfAUVRE, Nicolas, Histoire d'un journal local : les Alpes Mancelles de 1919 à 1939, dir. B. WACHÉ.

BARRÉ, Delphine, Henri-Irénée Marrou, acteur et témoin de son temps (19251945), dir. B. WACHÉ.

BARRÉ, Nathalie, Aspects de l'évolution socio-culturelle d'un village du sud de la Sarthe : Vaas entre 1848 et 1914, dir. B. WACHÉ.

BENAHMED, Laurence, Limplantation et l'influence de l'Ouesté́clair dans le département de la Sarthe, dir. B. WACHÉ.

BERMOND, Steve, La prison du Mans 1830-1875, dir. B. WACHÉ.

BEUVIER, Miguel, $L a$ " tempête du désert " dans le golfe arabo-persique (août 1990avril 1991), dir. B. WACHÉ.

BLANCHET, Antoine, Le camp de concentration de Pontmain (1914-1920), dir. B. WaCHÉ.

Buin, Sébastien, La Caisse d'Épargne du Mans de 1945 à 1965, dir. B. WACHÉ.

COchet, Nathalie, La vie culturelle au Mans pendant la Seconde Guerre mondiale, dir. B. WACHÉ.

Cochonneau, Gilles, Fêtes et cérémonies dans le canton de Château-du-Loir (1919-1939), dir. B. WACHÉ.

CouETtE, Marie-Pauline, La NEF. Entre Munich et Vichy, des équipes au ser- 
vice des valeurs humaines pour fonder la démocratie, dir. B. WACHÉ.

DelaPeYRIÈRE, Marie-José, La Société théosophique du Mans, branche “Persévérance »1913-1998, dir. B. WaCHÉ

Demeule, Fabienne, L'abbé Migorel, curé de Malétable et autres paroisses, dir. B. WACHÉ.

FERRÉ, Johannick, La commune de Paris vue à travers la presse sarthoise (mars-mai 1871), dir. B. WACHÉ.

FERRIER, Monique, Le décor dans la ville à travers le cinéma contemporain, dir. B. WACHÉ.

GENEAU, Virginie, L'évolution de l'esprit politique du Mans sous le régime de la monarchie de juillet, dir. B. WACHÉ.

GIROUX, Laurent, Les laïcs catholiques au Mans de Vatican Il au synode diocésain, dir. B. WaCHÉ.

HAVARD, Marinette, Le Bon Pasteur du Mans 1833-1871, dir. B. WaCHÉ.

JEANJEAN, Bertrand, La culture de masse dans l'œeuvre de Georges Duhamel, dir. B. WACHÉ.

JOUBERT, Séverine, Les paysans ornais dans la deuxième moitié du XIXe siècle, dir. B. WACHÉ.

LouIs, Axelle, Monseigneur de Bonfils, évêque du Mans (1898-1912), dir. B. WACHÉ.
MichaleCZeK, Igor, Jean-Marie Duchemin (1908-1988). D'une vocation chrétienne à un voyage au cour de l'Islam, dir. B. WACHÉ.

Mousset, Marielle, Le docteur Auguste Touchard. Sa vie publique de 1865 à 1900, dir. B. WACHÉ.

NORMAND, Julien, La réception de la pensée blondélienne dans la crise moderniste, dir. B. WaCHÉ.

PAINEAU, Rémi, L'information locale dans les quotidiens Maine Libre et Ouest-France en 1996, dir. B. Waché et C. MANIGAND.

Paulin, Cédric, $M^{g r}$ Sevin, prêtre intellectuel du Maine 1896-1967, dir. B. WACHÉ.

Rigaud, Sophie, Les sociétés de LibrePensée dans la Sarthe (1880-1905), dir. B. Waché.

RIvière, Sonia, La vie quotidienne au Mans pendant la première guerre mondiale, dir. B. WACHÉ.

SEBILO, Fabienne, La police municipale du Mans : organisation et activités entre 1900 et 1941, dir. B. WACHÉ.

Uzu, Véronique, Louis Basse, un notable manceau, dir. M. AuFFRET et B. WACHÉ.

Zahnoun, Rachid, L'évolution démographique, socio-économique et religieuse et ses rapports avec le comportement politique des Manceaux 19651995, dir. B. WACHÉ. 

Achevé d'imprimer

sur les presses du service reprographique de l'université Rennes 2 Haute-Bretagne

en juillet 2004

Imprimé en France 


\section{QUELQUES RÈGLeS DE PRÉSENTATION DES TEXTES À L’USAGE DES AUTEURS}

1 - Indiquer sous le titre de l'article la qualité et l'adresse institutionnelle de l'auteur Ex. : Xxxx Xxxxxx, maître de conférences en histoire contemporaine, université de X., groupe de recherches de rattachement.

2 - Remplacer le plus possible les majuscules (sauf pour les lettres initiales des noms propres) par des petites capitales. Ceci dans les titres, les sigles, les noms d'auteurs... Indiquer aussi les siècles en petites capitales : $\mathrm{XV}^{\mathrm{e}}$ siècle et non XVe siècle. Accentuer les majuscules.

3 - Abréviations : en user le moins possible

Pas d'abréviations pour le nom des revues, surtout pour les revues locales qui ne sont connues que des locaux.

Pour les dépôts d'archives :

- ne pas mettre ADIV ou AD 35 mais : Arch. dép. d'Ille-et-Vilaine, du Morbihan...;

- préférer Arch. nat. à AN;

- mettre les noms des bibliothèques en entier.

Développer les sigles entre parenthèses lors de leur première occurrence.

4 - Références bibliographiques

- mettre toujours et partout les prénoms et les noms (ne pas abréger le prénom par une initiale);

- utiliser les petites capitales pour le nom propre et non les majuscules, utiliser les minuscules pour le prénom; les séparer par des virgules.

Nom, Prénom, Titre de l'ouvrage en italique, Lieu d'édition, éditeur, " collection " (éventuellement), année, pages.

Une bibliographie peut être donnée en fin d'article si cela est nécessaire.

5 - Forme des notes

Préférer la forme traditionnelle (référence biblio. développée lors de sa première occurrence, abrégée ensuite) à la forme anglo-saxonne (nom, date, page).

6 - Titres et sous-titres

Il est préférable de s'en tenir à deux niveaux. Mais si cela s'avère nécessaire afin de ne pas obtenir de trop longues pages trop compactes, un troisième niveau d'intertitres peut être utilisé.

7 - Cartes et documents photographiques

- Les cartes au trait devront être d'une EXCELLENTE QUALITÉ GRAPHIQUE sans collage hasardeux ni légende manuscrite.

- Il est toujours préférable de FOURNIR LES ORIGINAUX, sinon, un fichier informatique au format .EPS ou .AI pour les cartes et plans ou .TIF pour les photographies. En aucun cas un ficher .JPG, de résolution trop basse.

8 - Autres questions non évoquées ici..

consulter la rédaction à l'adresse ci-dessous.

9 - Donnez une adresse mail et téléphone où l'on puisse vous joindre facilement au moment où l'on fait la mise en page de votre article.

Contact

Annie Antolne, Secrétaire de l'Association pour la publication des $A B P O$ université de Rennes 2, Département Histoire,

Place du Doyen-Henri-Le-Moal - 35043 RenNEs Cedex

e-mail : annie.antoine@uhb.fr

Les manuscrits sont à envoyer à l'adresse ci-dessus 